\title{
Feasibility of Wii Fit training to improve clinical measures of balance in older adults
}

\section{Kathleen A Bieryla Neil M Dold}

Biomedical Engineering Department, Bucknell University, Lewisburg, PA, USA
Correspondence: Kathleen Bieryla

One Dent Drive, Biomedical Engineering Department, Bucknell University,

Lewisburg, PA 17837, USA

$\mathrm{Tel}+|570577234|$

Fax + I 5705773659

Email k.bieryla@bucknell.edu
This article was published in the following Dove Press journal:

Clinical Interventions in Aging

22 June 2013

Number of times this article has been viewed

Background and purpose: Numerous interventions have been proposed to improve balance in older adults with varying degrees of success. A novel approach may be to use an off-the-shelf video game system utilizing real-time force feedback to train older adults. The purpose of this study is to investigate the feasibility of using Nintendo's Wii Fit for training to improve clinical measures of balance in older adults and to retain the improvements after a period of time.

Methods: Twelve healthy older adults (aged $>70$ years) were randomly divided into two groups. The experimental group completed training using Nintendo's Wii Fit game three times a week for 3 weeks while the control group continued with normal activities. Four clinical measures of balance were assessed before training, 1 week after training, and 1 month after training: Berg Balance Scale (BBS), Fullerton Advanced Balance (FAB) scale, Functional Reach (FR), and Timed Up and Go (TUG). Friedman two-way analysis of variance by ranks was conducted on the control and experimental group to determine if training using the Wii Balance Board with Wii Fit had an influence on clinical measures of balance.

Results: Nine older adults completed the study (experimental group $n=4$, control group $n=5$ ). The experimental group significantly increased their BBS after training while the control group did not. There was no significant change for either group with FAB, FR, and TUG.

Conclusion: Balance training with Nintendo's Wii Fit may be a novel way for older adults to improve balance as measured by the BBS.

Keywords: older adults, balance, training

\section{Introduction}

Every year, approximately one in three adults over the age of 65 years fall, and approximately one in two adults over the age of 80 years fall. ${ }^{1}$ Falls can lead to injuries including wrist sprains, bruises, and hip fractures, or even death. Even if a serious injury does not occur, the fear of falling can decrease the quality of life for older adults by inhibiting them from completing normal daily activities including going to the store, taking a bath, and bending down to pick an object up. ${ }^{2}$

Numerous interventions have been proposed to improve balance in older adults. These can range from strength training, to aerobic training, to tai chi, to task specific training. ${ }^{3-6}$ Results of these studies are inconsistent, with some showing significant positive influence on measures of balance while others had little or no effect. The use of video gaming systems for various types of rehabilitation has received high interest, particularly Nintendo's Wii. One game in particular, Nintendo's Wii Sports, has been used in recent studies that explored a variety of applications including improvement of visual perceptual processing, postural control, and mobility in an adolescent with 
cerebral palsy, ${ }^{7}$ decreased fall risk in a single elderly nursing home resident, ${ }^{8}$ and improvement of mean motor function in patients poststroke. ${ }^{9}$

Combining real-time force feedback with a video game may be one way to improve measures of balance in older adults. An expensive, custom made, video game system that uses the person's center of pressure to control a game has shown promise for balance rehabilitation in people with spinal cord and traumatic brain injuries. ${ }^{10,11}$ Using an off the shelf system such as Nintendo's Wii Balance Board may be a novel and inexpensive way to improve balance in older adults through training. The Wii Balance Board is similar to a force platform as it can monitor the center of pressure of a person, but it is more user friendly. ${ }^{12}$ Numerous video games exist to interact with the Wii Balance Board. One game that has been used in prior balance studies is Wii Fit. This game allows users to complete various training modes such as yoga, strength training, aerobics, and balance games. These training modes are similar to previous balance training interventions, but provide real-time force feedback. Wii Fit training has been examined in case studies ${ }^{13,14}$ and noncontrolled pilot studies. ${ }^{15-17}$ Few studies have used a control group when examining the effect of training on the Wii Balance Board to improve measures of balance in healthy older adults. One study utilized older adults to compare traditional physical therapy to therapy using Wii Fit and saw improvements with both groups in the Berg Balance Scale (BBS), though this was not a strict control group without any intervention. ${ }^{18}$ Williams et al saw an improvement in the BBS in a Wii Fit intervention group after a 12 week training regime, while there was no improvement in the control group. ${ }^{19}$ In a larger randomized controlled study that involved 28 older adults in a Wii Fit intervention group and 30 older adults in a control group, significantly larger pre- to posttraining changes were seen in the Timed Up and Go test (TUG), 30 second repeated Chair Stand Test, and the short Falls Efficacy ScaleInternational for the Wii group. ${ }^{20}$

Currently, there is no controlled study that examines retention of Wii Fit training in older adults on clinical measure of balance. Therefore, the purpose of this study is to investigate the feasibility of using the Wii Balance Board with Wii Fit to improve clinical measures of balance in older adults and retain the improvements after a period of time. Four commonly used clinical balance tests were measured before and after an intervention including the BBS, Fullerton Advanced Balance Scale (FAB), Functional Reach (FR), and TUG. It is hypothesized that older adults who train with Wii Fit will increase their BBS, FAB, FR, and decrease their TUG time, and retain the improvements, while those who do not complete the training will not improve.

\section{Methods}

Twelve healthy older adults (two male and ten female) were recruited from a local senior living community ranging in age from 70-92 years old (81.5 \pm 5.5 years, mean \pm SD). All participants lived independently, were able to stand unassisted for 30 minutes, and walk a minimum of 6 meters without aid. Participants were not screened for any neurologic or vestibular disorders prior to the study. The study was approved by Bucknell University's Institutional Review Board and written consent was obtained from all participants prior to participation.

The experiment employed a pretest-posttest control group design. Participants were randomly assigned to either an experimental $(82.5 \pm 1.6$ years $)$ or control group $(80.5 \pm 7.8$ years) while maintaining one male in each group (Figure 1). Mann-Whitney $U$-tests showed the groups did not significantly differ in age, weight, or height $(P>0.05)$. In the experimental group, one participant discontinued the training due to personal reasons, and one participant was unable to attend the second follow-up session. In the control group, one person was lost at the first follow-up due to scheduling conflicts. Each group completed a series of clinical measures of balance before and after an intervention. Participants in the experimental group trained using the Wii Balance Board with Wii Fit while the control group continued their normal daily activities.

Four different clinical measures of balance were assessed: BBS, FAB scale, FR, and TUG. The BBS consists of 14 items

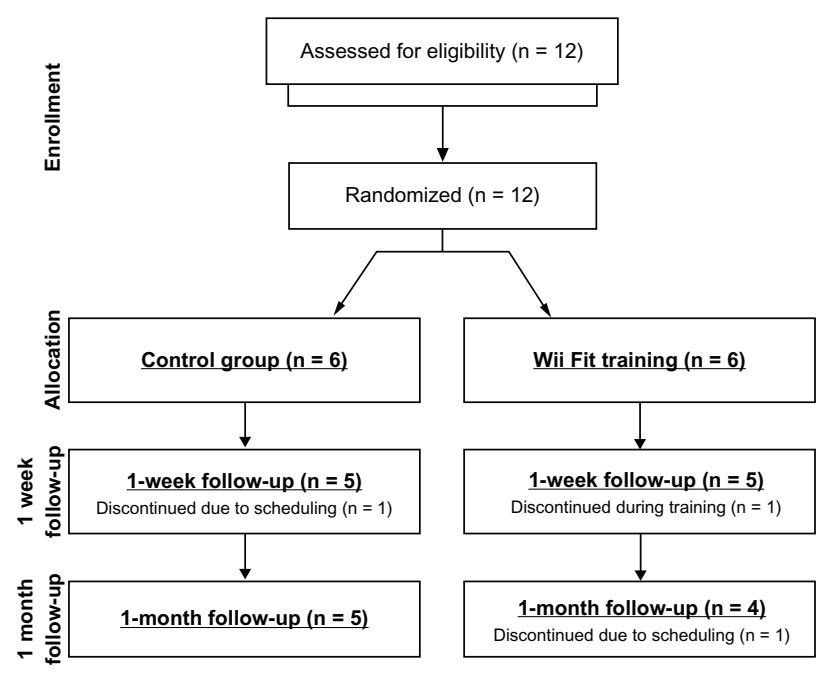

Figure I CONSORT diagram to show enrollment and retention of participants through the study.

Abbreviation: CONSORT, Consolidated Standards Reporting of Trials. 
ranging from standing with eyes shut to rising from a chair. ${ }^{21}$ Each item is scored on a $0-4$ scale (maximum score $=56$ ) with scores lower than 45 indicating an individual with a high risk for falls. ${ }^{22}$ The FAB is similar to the BBS as it is used to assess balance, but uses more dynamic tasks in an attempt to better discriminate adults with balance problems that may be higher functioning. The FAB consists of ten items ranging from a tandem walk to a two footed jump. ${ }^{23}$ Each item is scored on a $0-4$ scale with scores lower than 25 indicating an individual with a high risk for falls. ${ }^{24}$ Due to inconsistencies in testing one item, reactive postural control, it was removed and the FAB test was completed with nine items (maximum score $=36$ ). The FR test was performed with the participant raising their right arm parallel to the floor and leaning forward as far as possible without taking a step or lifting their heels. ${ }^{25}$ Three trials were recorded and the total distance reached averaged. A reach of less than $15.24 \mathrm{~cm}$ may indicate an individual with a high risk for falls. ${ }^{26}$ Finally, the TUG consists of the participant rising from a chair, walking 6 meters at a normal pace, turning around, and returning to the chair while being timed. ${ }^{27}$ Three trials were completed and the times averaged. A TUG time of greater than 14 seconds may indicate an individual with a high risk for falls. ${ }^{27}$ All assessments were conducted by both KAB and NMD, who were not blinded to the group allocation.

After completing the balance tests, participants in the experimental group completed training using the Wii Balance Board with Wii Fit (Figure 2). Training occurred three times a week (Monday, Wednesday, and Friday) for 3 weeks. Each training session was one-on-one with a supervisor, lasted approximately 30 minutes, and was the same for all participants. All training was supervised, but no physical assistance was given to any participant. Chairs were placed in front of and to the left and right of the Balance Board for safety in case a participant lost balance. The intervention consisted of a series of exercises and activities chosen from the yoga (half moon, chair, warrior), aerobic (torso twists), and balance games (soccer heading, ski jump) modes. The specific order of the training was half moon, chair, warrior, torso twists, followed by two rounds of the soccer heading and ski jump, and finished with a repeat of the half moon, chair, warrior, and torso twists. Although the training program was not varied between individuals or within individuals throughout the intervention, all participants were challenged consistently. All participants in the experimental group were novices with Wii Fit prior to training, and as such there was room for improvement after beginning training. The consistent challenge was observed with the participants

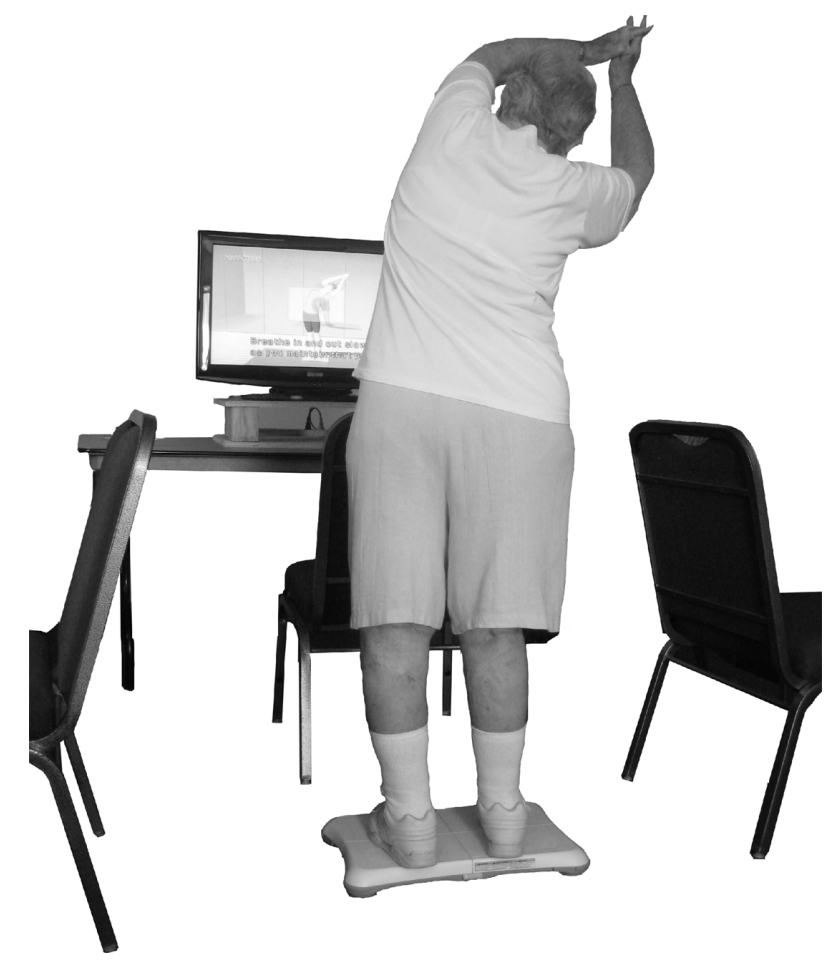

Figure 2 Participant performing the half moon pose during one training session.

commenting on their improved scores during the balance games as training progressed. Participants were informed that the scores from the two balance games would not be used in analysis, and were encouraged to try their best during the games. Participants in the control group continued with their normal daily activities. After the conclusion of the 3 week intervention on a Friday, all participants returned the following week for their first postintervention testing, referred to as 1 week postintervention. Participants completed the series of balance tests in the same manner prior to the intervention. After the intervention, participants were instructed to continue with their normal daily activities. Three weeks after the 1 week postintervention testing, a second testing was conducted, referred to as 1 month postintervention. Again, participants completed the series of balance tests in the same manner prior to the intervention to determine if any improvements were retained.

Clinical measures of balance before the intervention were compared to 1 week and 1 month after the intervention. Nonparametric statistical analysis was conducted due to the inability to assume normal distribution with the small sample size. Mann-Whitney $U$-tests were conducted on preintervention measures of balance between the control and experimental group. Descriptive statistics (median and interquartile range) for the control and experimental group were calculated for all dependent measures. Friedman two-way 
analysis of variance by ranks was conducted on the control and experimental group to determine if training using the Wii Balance Board with Wii Fit had an influence on clinical measures of balance. Upon significance, one-sided treatment versus control multiple comparisons were conducted. ${ }^{28}$ Statistical analysis was completed in Minitab 16.2.3 (Minitab Inc, State College, PA, USA) and statistical significance was set at $P<0.05$.

\section{Results}

Preintervention scores did not significantly differ between the experimental and control group for $\operatorname{BBS}(P=0.711)$, FAB $(P=0.621)$, FR $(P=0.902)$, and TUG $(P=0.713)$. All participants who completed Wii Fit training $(n=5)$ attended all nine individual training sessions.

BBS scores significantly increased for participants who completed the training using Wii Fit $(P=0.037)$ (Table 1). Post hoc analysis indicated a significant increase from preintervention to the 1 month postintervention $(P<0.046)$ but not a significant increase from preintervention to 1 week postintervention $(P>0.102)$. Although there was not a significant change preintervention to 1 week postintervention, all but one participant increased their BBS after Wii Fit training. BBS scores did not significantly change for participants in the control group $(P=0.161)$.

FAB scores did not significantly increase for participants who completed the training using Wii Fit $(P=0.529)$ (Table 1). In addition, FAB scores did not significantly change for participants in the control group $(P=0.946)$.

FR scores did not significantly increase for participants who completed the training using Wii Fit $(P=0.779)$ (Table 1). In addition, FR scores did not significantly change for participants in the control group $(P=0.949)$.

TUG scores did not significantly decrease for participants who completed the training using Wii Fit $(P=0.174)$ (Table 1). In addition, TUG scores did not significantly change for participants in the control group $(P=0.549)$.

\section{Discussion}

The purpose of this study was to investigate the feasibility of using Nintendo's Wii Balance Board with Wii Fit to improve clinical measures of balance in older adults and to retain the improvements after a period of time. After 3 weeks of Wii Fit training, older adults were able to improve their BBS score where older adults who did not complete the training did not improve their BBS. Post hoc analysis indicated a significant difference between preintervention and 1 month postintervention for the experimental group. Minimal detectable change with $95 \%$ confidence for the BBS in healthy older adults has been identified as four. ${ }^{29}$ Of the four experimental group participants who completed the study, two obtained the BBS minimal detectable change of four from preintervention to 1 month postintervention. There was no significant improvement in FAB, FR, or TUG for either group.

The BBS results from the current study are in agreement with the few studies that have examined the efficacy of training using the Wii Balance Board to improve measures of balance. Eight women who completed a 10 week training program using Wii Fit were able to significantly improve some measures of balance immediately following the training, including unilateral stance time, but not others such as TUG. ${ }^{17}$ Another similar study compared groups of older adults who completed traditional physical therapy balance training, Wii Fit training, or a combined physical therapy balance training with Wii Fit training. ${ }^{18}$ All training groups were able to increase their BBS after 4 weeks of training. There are distinct differences between the current study and past studies. First, neither of the previous studies utilized a group that did not complete some form of training. Without a control group, the changes in balance measures cannot be directly attributed to Wii Fit training. Also, the training completed with Wii Fit was distinctly different in all studies. Nitz et a $1^{17}$ allowed participants to choose their own exercises for the training program while Bateni ${ }^{18}$ limited the training to only three balance games. The current study used the

Table I Median of outcome variables preintervention, I week postintervention, and I month postintervention for both the control and experimental group

\begin{tabular}{|c|c|c|c|c|c|c|}
\hline & \multicolumn{3}{|l|}{ Control } & \multicolumn{3}{|l|}{ Experimental } \\
\hline & Pre $(n=5)$ & $\begin{array}{l}\text { I week post } \\
(n=5)\end{array}$ & $\begin{array}{l}\text { I month post } \\
(n=5)\end{array}$ & Pre $(n=5)$ & $\begin{array}{l}\text { I week post } \\
(n=5)\end{array}$ & $\begin{array}{l}\text { I month post } \\
(n=4)\end{array}$ \\
\hline BBS & $51(44-54)$ & $54(47-55.5)$ & $54(43-54.5)$ & $50(47.5-5 \mid .5)$ & $52(49.5-53.5)$ & $53(52-54)^{*}$ \\
\hline FAB & $31(19-33)$ & $25(18-36.5)$ & $32(17.5-33)$ & $27(21.5-29.5)$ & $27(23.5-31.5)$ & $28.5(24-30.7)$ \\
\hline $\mathrm{FR}(\mathrm{cm})$ & $28.7(|8.6-3| .8)$ & $25(17.3-32.5)$ & $28.7(I 5.5-30.6)$ & $24.3(22.3-32.7)$ & $26(18.7-28.5)$ & $23.5(22.3-29.7)$ \\
\hline TUG (s) & $10.8(9.2-19.2)$ & $10.9(9.2-17.6)$ & $10.1(9.3-17.1)$ & $12.8(\mid 2.2-14.9)$ & $11.9(11.1-15.1)$ & II.2(I0.6-14.3) \\
\hline
\end{tabular}

Notes: Data are presented as median with interquartile range. *Represent significant $(P<0.05)$ difference from before training measurement. Abbreviations: BBS, Berg Balance Scale; FAB, Fullerton Advanced Balance Scale; FR, Functional Reach; TUG, Timed Up and Go. 
same training protocol for all participants and was a mix of the three training modes including yoga, aerobics, and balance games. This was chosen to provide experimental control, improving internal validity. Neither of the previous studies examined the retention of any improvements from the training. Finally, no other study has examined the effect of force feedback training on FAB scores.

In the current study, two of the four participants in the experimental group increased their FAB score after Wii Fit training 1 week postintervention, but additionally, two of five participants increased their FAB with no intervention. The FAB test has some items that overlap with the BBS, but the FAB consists of more dynamics tasks such as stepping up and over a six inch high bench, a tandem walk, and a two footed jump. ${ }^{23}$ For example, all of the participants in the Wii Fit training group began with a score of two or below (out of four) for the two footed jump. Four of five participants had no change in their score after training while one subject decreased their score by one. On the same task, two participants in the control group increased their score by one, two participants had no change in score, and one participant decreased their score by two. As such, Wii Fit training may not improve the dynamic tasks such as these because participants must keep their feet on the Balance Board at all times. This static foot position may not translate to the more dynamic activities included in the FAB.

FR scores also did not improve after training with the Wii Fit. This was unexpected as Wii Fit forces participants to be more aware of their center of pressure and limit of stability with real-time visual feedback. Interventions, such as tai chi, that allow participants to be aware of their limit of stability and center of mass location on their own, without the real-time visual feedback, have improved FR in healthy older adults ${ }^{30}$ and transitionally frail older adults. ${ }^{31}$ One balance game in particular, ski jump, has participants lean as far forward as they can and then quickly straighten the body. Participants may not have fully challenged themselves during training for fear of falling forward. This may be one possible reason that training did not translate into improvement in FR, though fear of falling was not assessed in this study.

Significant improvement was not shown in TUG, and this may be due to a ceiling effect on older adults who are high functioning. ${ }^{32}$ Six out of nine participants who completed the study had an initial TUG within one standard deviation of the mean for normal healthy older adults aged $80-89$ years old. ${ }^{33}$ No background on prior falls was collected from the participants, so it is unknown if the participants with the higher initial TUG were different from participants with lower initial times with respect to the number of prior falls. Training with Wii Fit may be advantageous for those with high initial TUG times indicating a higher risk for falls than relatively healthy older adults. ${ }^{27,34}$ For example, a case study on a single patient poststroke showed that after four training sessions with Wii Fit, the patient was able to decrease their TUG by 10 seconds and also increase their FR and BBS. ${ }^{14}$ As stated earlier, tasks completed during training with the Balance Board require control of the center of pressure. This training may not translate to a mobility task such as TUG. Lack of improvement in dynamic measures was also seen in Toulotte et al where older adults training with the Wii Fit were able to improve only in the static conditions of the Tinetti test. ${ }^{35}$

The possibility of using the Wii Balance Board with Wii Fit to improve balance in older adults is appealing for many reasons. First, participants tend to enjoy what they are doing. Patients poststroke enjoyed therapy with the Wii Balance Board and thought it to be more challenging and fun compared to regular therapy. ${ }^{36}$ This was also observed in the current study. Although no formal survey following training was collected, participants stated their enjoyment, particularly with the two balance games. Another benefit to using the Wii Balance Board with Wii Fit for balance training is the low cost for the entire system which retails for under US\$250. One of the first studies to explore the use of video game based exercise for balance rehabilitation designed their own system. ${ }^{10}$ The system consisted of a pressure mat and custom designed software with an estimated cost of US\$8700. The low cost of the commercially available system would allow people to continue the training in the comfort of their own home without supervision. Therefore, the feasibility of implementing this training program at home is high. This allows potential for long term adherence to a training program outside of the study, and may lead to longer retention of any improvement from the training sessions.

There are several limitations with this study. First, the final sample size is small $(\mathrm{n}=9)$. Though the results are promising, further research is needed before the results can be generalized to the population at large. The lack of significant changes in FAB, FR, and TUG may be due to insufficient number of subjects needed to detect a change. Second, the current study examined retention only 1 month after training was complete. For a training program to be beneficial, any improvements need to remain after training has stopped. Further research is needed to determine how long improvements would remain. Additionally, the 3 week training may not have been long enough to detect initial 
improvements in FAB, FR, and TUG. An improvement in FAB scores for healthy older adults was seen after an 8 week traditional exercise intervention. ${ }^{37} \mathrm{~A}$ longer intervention may have resulted in changes in the other balance measures. Furthermore, the activities of the participants outside the study were not strictly controlled. Participants were told to continue with their normal daily routine and were not required to stop any activity. This may have led to improvements in balance not controlled for in the study. Finally, the fitness level prior to entering the study may have influenced the results, though initial balance measurements prior to the start of the intervention indicated no significant difference between the experimental and control groups.

\section{Conclusion}

In conclusion, training older adults using Nintendo's Balance Board and Wii Fit was shown to improve BBS scores 1 month after the intervention concluded. Further research is needed to examine the lasting effects of the improvement and the ideal training program. Expanding the training time and sample size may result in changes in the other balance measures and should be explored. Wii Fit training may be a novel and fun way for older adults to improve some clinical measures of balance in a low cost manner at home.

\section{Disclosure}

The authors declare no conflict of interest in this work.

\section{References}

1. Tinetti ME, Speechley M, Ginter SF. Risk factors for falls among elderly persons living in the community. N Engl J Med. 1988;319(26):1701-1707.

2. Li F, Fisher KJ, Harmer P, McAuley E, Wilson NL. Fear of falling in elderly persons: Association with falls, functional ability, and quality of life. J Gerontol B Psychol Sci Soc Sci. 2003;58(5):P283-P290.

3. Buchner DM, Cress ME, de Lateur BJ, et al. The effect of strength and endurance training on gait, balance, fall risk, and health services use in community-living older adults. J Gerontol A Biol Sci Med Sci. 1997;52(4):M218-M224.

4. Faber MJ, Bosscher RJ, Chin A, Paw MJ, van Wieringen PC. Effects of exercise programs on falls and mobility in frail and pre-frail older adults: A multicenter randomized controlled trial. Arch Phys Med Rehabil. 2006;87(7):885-896.

5. Woo J, Hong A, Lau E, Lynn H. A randomised controlled trial of tai chi and resistance exercise on bone health, muscle strength and balance in community-living elderly people. Age Ageing. 2007;36(3):262-268.

6. Bieryla KA, Madigan ML, Nussbaum MA. Practicing recovery from a simulated trip improves recovery kinematics after an actual trip. Gait Posture. 2007;26(2):208-213.

7. Deutsch JE, Borbely M, Filler J, Huhn K, Guarrera-Bowlby P. Use of a low-cost, commercially available gaming console (Wii) for rehabilitation of an adolescent with cerebral palsy. Phys Ther. 2008;88(10): 1196-1207.

8. Clark R, Kraemer T. Clinical use of Nintendo Wii bowling simulation to decrease fall risk in an elderly resident of a nursing home: a case report. J Geriatr Phys Ther. 2009;32(4):174-180.
9. Saposnik G, Teasell R, Mamdani M, et al; Stroke Outcome Research Canada (SORCan) Working Group. Effectiveness of virtual reality using wii gaming technology in stroke rehabilitation: A pilot randomized clinical trial and proof of principle. Stroke. 2010;41(7):1477-1484.

10. Betker AL, Szturm T, Moussavi ZK, Nett C. Video game-based exercises for balance rehabilitation: A single-subject design. Arch Phys Med Rehabil. 2006;87(8):1141-1149.

11. Betker AL, Desai A, Nett C, Kapadia N, Szturm T. Game-based exercises for dynamic short-sitting balance rehabilitation of people with chronic spinal cord and traumatic brain injuries. Phys Ther. 2007;87(10):1389-1398.

12. Clark RA, Bryant AL, Pua Y, McCrory P, Bennell K, Hunt M. Validity and reliability of the nintendo wii balance board for assessment of standing balance. Gait Posture. 2010;31(3):307-310.

13. Pigford T, Andrews A. Feasibility and benefit of using the Nintendo Wii Fit for balance rehabilitation in an elderly patient experiencing recurrent falls. J Stud Phys Ther Res. 2010;2(1):12-20.

14. Sugarman H, Weisel-Eichler A, Burstin A, Brown R. Use of the Wii Fit system for the treatment of balance problems in the elderly: A feasibility study. In: Virtual Rehabilitation International Conference, 2009. Haifa, Israel: IEEE; 2009:111-116.

15. Agmon M, Perry CK, Phelan E, Demiris G, Nguyen HQ. A pilot study of Wii Fit exergames to improve balance in older adults. $J$ Geriatr Phys Ther. 2011;34(4):161-167.

16. Bainbridge E, Bevans S, Keeley B, Oriel K. The effects of the Nintendo Wii Fit on community-dwelling older adults with perceived balance deficits: A pilot study. Phys Occup Ther Geriatr. 2011;29(2): 126-135.

17. Nitz JC, Kuys S, Isles R, Fu S. Is the Wii Fit a new-generation tool for improving balance, health and well-being? A pilot study. Climacteric. 2010;13(5):487-491.

18. Bateni H. Changes in balance in older adults based on use of physical therapy vs the Wii Fit gaming system: A preliminary study. Physiotherapy. 2012;98(3):211-216.

19. Williams MA, Soiza RL, Jenkinson AM, Stewart A. EXercising with Computers in Later Life (EXCELL)-pilot and feasibility study of the acceptability of the Nintendo ${ }^{\circledR}$ Wii Fit in community-dwelling fallers. BMC Res Notes. 2010;3(1):238.

20. Jorgensen MG, Laessoe U, Hendriksen C, Nielsen OBF, Aagaard P. Efficacy of nintendo wii training on mechanical leg muscle function and postural balance in community-dwelling older adults: A randomized controlled trial. J Gerontol A Biol Sci Med Sci. Epub October 31, 2012.

21. Berg KO, Wood-Dauphinee SL, Williams JI, Maki B. Measuring balance in the elderly: Validation of an instrument. Can J Public Health. 1992;83 Suppl 2:S7-S11.

22. Bogle Thorbahn LD, Newton RA. Use of the berg balance test to predict falls in elderly persons. Phys Ther. 1996;76(6):576-583.

23. Rose DJ, Lucchese N, Wiersma LD. Development of a multidimensional balance scale for use with functionally independent older adults. Arch Phys Med Rehabil. 2006;87(11):1478-1485.

24. Hernandez D, Rose DJ. Predicting which older adults will or will not fall using the fullerton advanced balance scale. Arch Phys Med Rehabil. 2008;89(12):2309-2315.

25. Duncan PW, Weiner DK, Chandler J, Studenski S. Functional reach: A new clinical measure of balance. J Gerontol. 1990;45(6):M192-M197.

26. Duncan PW, Studenski S, Chandler J, Prescott B. Functional reach: Predictive validity in a sample of elderly male veterans. $J$ Gerontol. 1992;47(3):M93-M98.

27. Shumway-Cook A, Brauer S, Woollacott M. Predicting the probability for falls in community-dwelling older adults using the timed up and go test. Phys Ther. 2000;80(9):896-903.

28. Hollander M, Wolfe DA. Nonparametric Statistical Methods, 2nd ed. New York: John Wiley and Sons, Inc; 1999.

29. Donoghue D, Stokes EK. How much change is true change? the minimum detectable change of the berg balance scale in elderly people. J Rehabil Med. 2009;41(5):343-346. 
30. Li F, Harmer P, Fisher KJ, et al. Tai chi and fall reductions in older adults: A randomized controlled trial. J Gerontol A Biol Sci Med Sci. 2005;60(2):187-194.

31. Wolf SL, Sattin RW, Kutner M, O’Grady M, Greenspan AI, Gregor RJ. Intense tai chi exercise training and fall occurrences in older, transitionally frail adults: A randomized, controlled trial. J Am Geriatr Soc. 2003;51(12):1693-1701.

32. Boulgarides LK, McGinty SM, Willett JA, Barnes CW. Use of clinical and impairment-based tests to predict falls by community-dwelling older adults. Phys Ther. 2003;83(4):328-339.

33. Steffen TM, Hacker TA, Mollinger L. Age-and gender-related test performance in community-dwelling elderly people: Six-minute walk test, berg balance scale, timed up and go test, and gait speeds. Phys Ther. 2002;82(2):128-137.
34. Trueblood PR, Hodson-Chennault N, McCubbin A, Youngclarke D Performance and impairment-based assessments among community dwelling elderly: Sensitivity and specificity. Journal of Geriatric Physical Therapy. 2001;24(1):2-6.

35. Toulotte C, Toursel C, Olivier N. Wii fit ${ }^{\mathbb{R}}$ training vs. adapted physical activities: Which one is the most appropriate to improve the balance of independent senior subjects? A randomized controlled study. Clin Rehabil. 2012.

36. Lange B, Flynn S, Proffitt R, Chang CY, Rizzo AS. Development of an interactive game-based rehabilitation tool for dynamic balance training. Top Stroke Rehabil. 2010;17(5):345-352.

37. Westlake KP, Culham EG. Sensory-specific balance training in older adults: Effect on proprioceptive reintegration and cognitive demands. Phys Ther. 2007;87(10):1274-1283.
Clinical Interventions in Aging

\section{Publish your work in this journal}

Clinical Interventions in Aging is an international, peer-reviewed journal focusing on evidence-based reports on the value or lack thereof of treatments intended to prevent or delay the onset of maladaptive correlates of aging in human beings. This journal is indexed on PubMed Central, MedLine, the American Chemical Society's 'Chemical Abstracts
Dovepress

Service' (CAS), Scopus and the Elsevier Bibliographic databases. The manuscript management system is completely online and includes a very quick and fair peer-review system, which is all easy to use. Visit http://www.dovepress.com/testimonials.php to read real quotes from published authors.

Submit your manuscript here: http://www.dovepress.com/clinical-interventions-in-aging-journal 